\title{
Study of the combined muffler effectiveness
}

\author{
Kristina Chichvarina* and Sergey Smirnov \\ B M STU, Power Engineering Department, 105005, M oscow, Russian Federation
}

\begin{abstract}
The paper presents the research of the spectral characteristics of a combined reflection and absorption muffler with a different ratio of dissipative elements and reactive components for a given muffler volume.
\end{abstract}

\section{Introduction}

The intensive grow th of the vehicle fleet predetermines the growth of the significance of the problem of noise pollution of the human environment. One of the most active sources of vehicle noise is the internal combustion engine (ICE). The noise of the internal combustion engine, taking into account the mechanisms of formation of its individual components, is divided into aerodynamic and structural. A erodynamic noise, in terms of its level, significantly exceeds structural noise. However, the problem of its reduction is currently being successfully solved by selecting the volume and structure of mufflers in the intake and exhaust systems, while the aerodynamic resistance of the muffler should be minimal [1-6].

According to the principle of operation, mufflers are divided into two main groups reactive and dissipative mufflers. In reactive mufflers, noise reduction behind the muffler is achieved mainly due to the reflection of waves incident on it with subsequent interference; in dissipative ones - due to the transformation of the sound energy of the incident waves into heat in the sound-absorbing material located in the muffler [2]. The gas flow in such mufflers is usually directed al ong the surface of the absorber. In most cases, their hydraulic resistance is low. Silencers, for which both reflection and dissipation are essential, are called combined.

The effective operation of absorbing structures in a wide frequency range is ensured when the thickness of the absorber layers placed on them is of the order of a quarter of the wavelength of the sound being damped. To reduce the level of low-frequency noise, the required dimensions of the absorbing elements become too large and the use of reactive silencers turns out to be more effective.

Reactive noise mufflers are usually made in the form of a system of expansion and resonance chambers, connected to each other and to the volume of the air duct using pipes and holes [3]. Combined silencers in the low-frequency region act as reflectors, and in the high-frequency region - as sound absorbers.

* Corresponding author: Christina160195@gmail.com 


\section{The investigated combined muffler}

In this work, the acoustic characteristics of a combined noise muffler were investigated, consisting of two expansion chambers, connected by pipes, and dissipative elements. In this case, three of its configurations were considered: mufflers in which the volume fraction of the dissipative part is $72.1 \%$ (Figure 1), $49.9 \%$ (Figure 2) and 35\% (Figure 3) of a given volume. The structures themselves have two expansion chambers, while the length of the small chamber is $2 / 3$ of the length of the large chamber, in addition, the muffler contains three dissipative elements, which are part of the inlet, connecting and outlet pipes, while the corresponding parts of these pipes are perforated, and the end parts of these pipes perform the function of quarter-wave resonators. The total volume of the muffler under consideration is 17 liters, taken from the indicators of the analysis of the market for noise mufflers for a two-liter internal combustion engine.

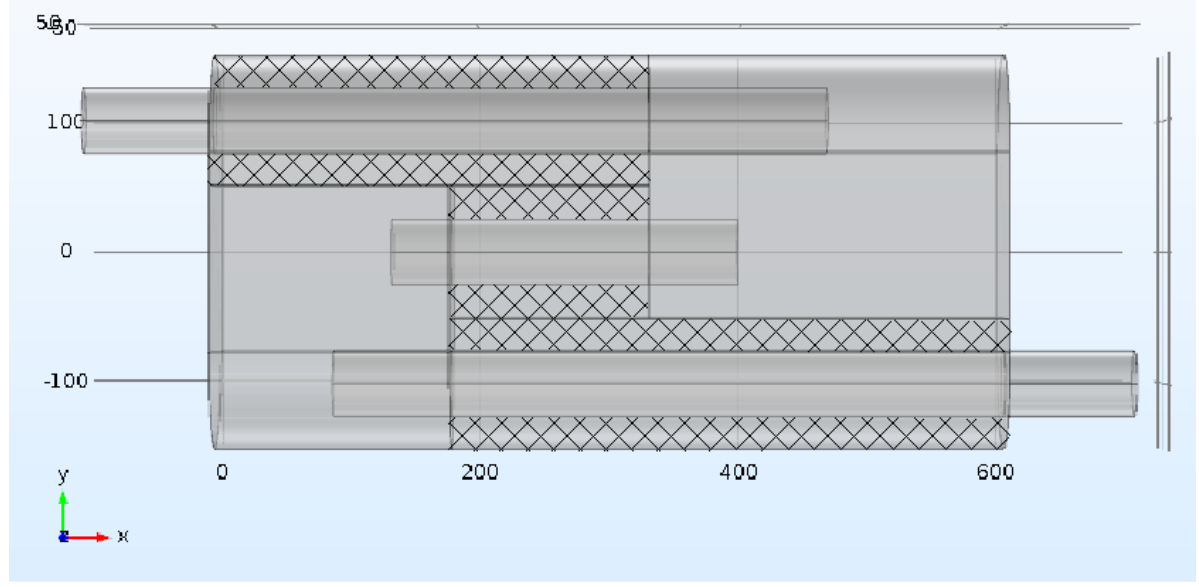

Fig. 1. Configuration in which the volume fraction of the dissipative part is $72.1 \%$.

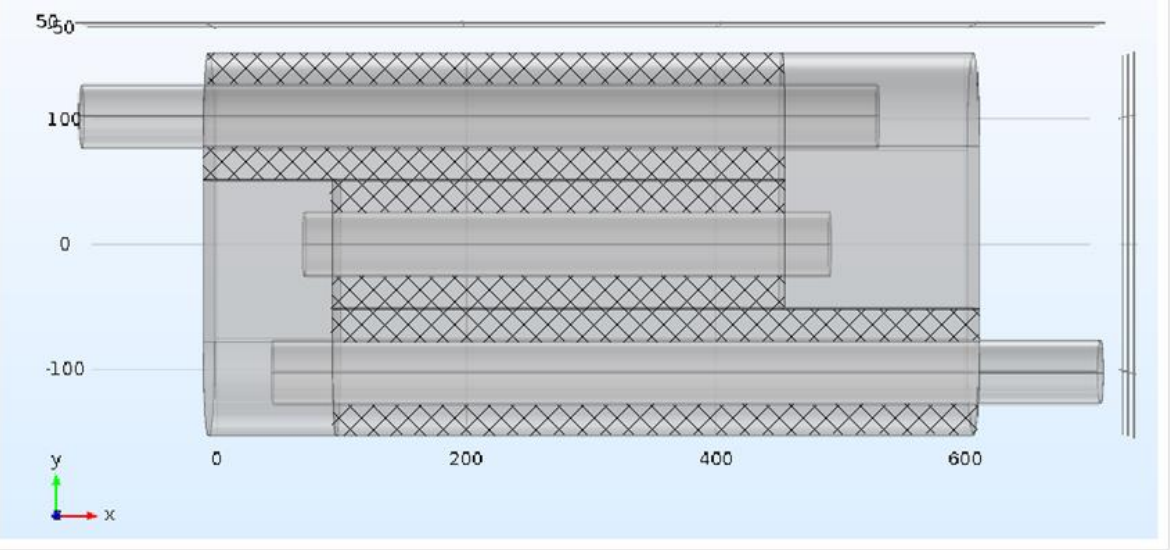

Fig. 2. Configuration in which the volume fraction of the dissipative part is $49.9 \%$. 


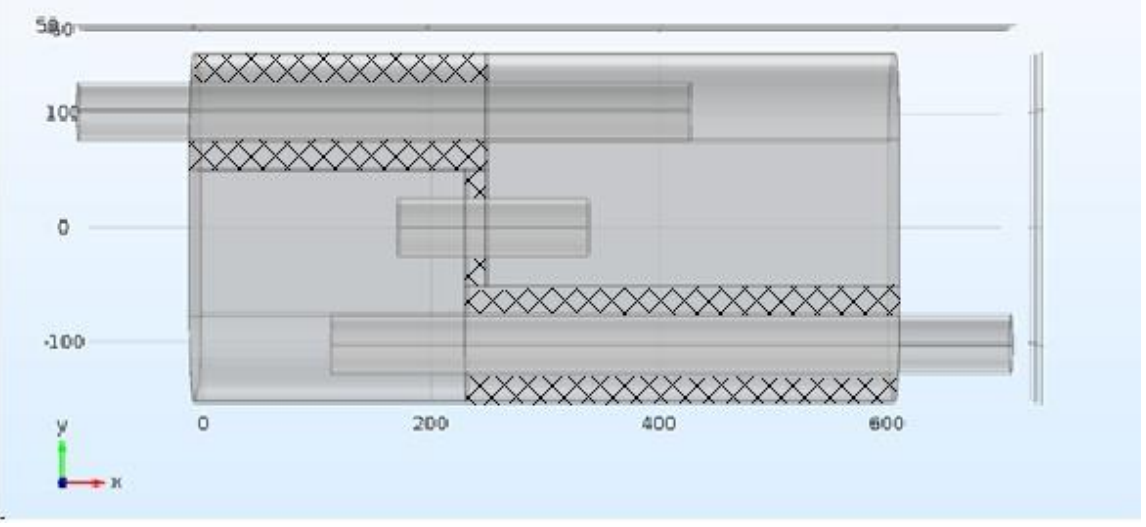

Fig. 3. Configuration in which the volume fraction of the dissipative part is $35 \%$.

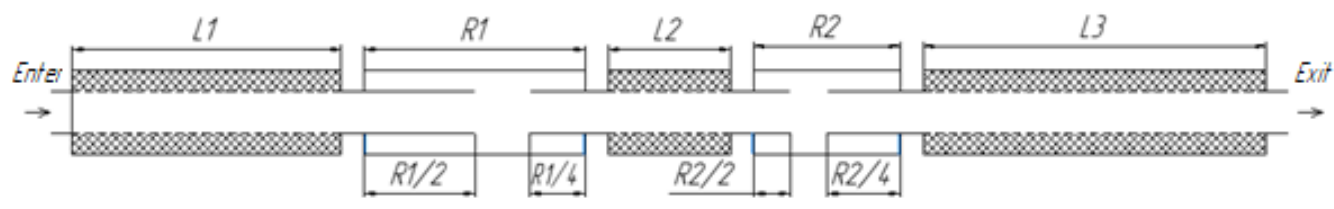

Fig. 4. Equivalent acoustic scheme of the muffler.

In Figure 4 shows the equival ent acoustic scheme of the considered muffler. Initially, the exhaust gases enter the first lining channel of length $L 1$, which has a perforated pipe and a cavity filled with a SAM. For each design of the muffler, the lengths of the channels and chambers are different, since it depends on the ratio of dissipative and reactive el ements in a given volume of the muffler, respectively; this applies to the following values: $R 1, L 2, R 2$, and $L 3$. Further, the gas flow passes through the first expansion chamber with dimensions $\mathrm{R} 1$, into which the first branch pipe enters to the length $R 1 / 2$, and the second to $R 1 / 4$. This type of camera has reactive properties. Through the second branch pipe, exhaust gases enter the second lining channel of length $L 2$, which has dissipative properties, similar to the first. The next element in the acoustic path is the second expansion chamber, which has a length $R 2$. In it, as in the first chamber, connecting pipes entering the chamber is located, but only the flow enters the chamber through a pipe with a length of $R 2 / 2$ and moves into the next lining channel through a pipe with a length of $R 2 / 4$. The final element in this acoustic path is the third lining duct of length $L 3$ (which has similar properties to the ducts $L 1, L 2$ ), through which exhaust gases enter the exhaust system.

\section{Numerical calculation of the noise muffler}

The calculation of the considered silencer was carried out by the finite element method in the Comsol Multiphysics software package. In addition to the geometric dimensions of the muffler and the speed of sound, the properties of the sound-absorbing material were set: the density of its packing, $\rho=120 \mathrm{~kg} / \mathrm{m}^{3}$, and the average fiber diameter $d_{\mathrm{av}}=12 \mu \mathrm{m}$. The pressure at the inlet of the muffler in the calculations was $2000 \mathrm{~Pa}$, which corresponds to the sound pressure level $L=160 \mathrm{~dB}$.

In the calculations, to assess the acoustic efficiency of the muffler, we used such a parameter as transmission loss TL (Transmission Loss), which is determined through the 
logarithm of the ratio of sound powers at the entrance and exit of the muffler: $T L=10 \lg \left(\mathrm{W}_{\text {in }} / \mathrm{W}_{\text {out }}\right)$.

In the course of the work, transmission losses of mufflers with a different volumetric distribution of reactive and dissipative parts were determined. The corresponding graphical dependencies are shown in Figure 5, where number 1 denotes the spectrum with a fraction of the dissipative part in the muffler volume of $35 \%$, number $2-49.9 \%$, number $3-72.1 \%$.

Calculations have shown that a muffler with a higher percentage of SA M works better at high frequencies, and with a low content - at low frequencies. In general, any of the investigated configurations are, on average, capable of reducing SPL to 50-60 dB from the initial level equal to $160 \mathrm{~dB}$. A t the same time, it can be noted that where the large expansion chamber has lower efficiency, the small chamber becomes effective and vice versa. In general, the third silencer configuration has the greatest acoustic efficiency.

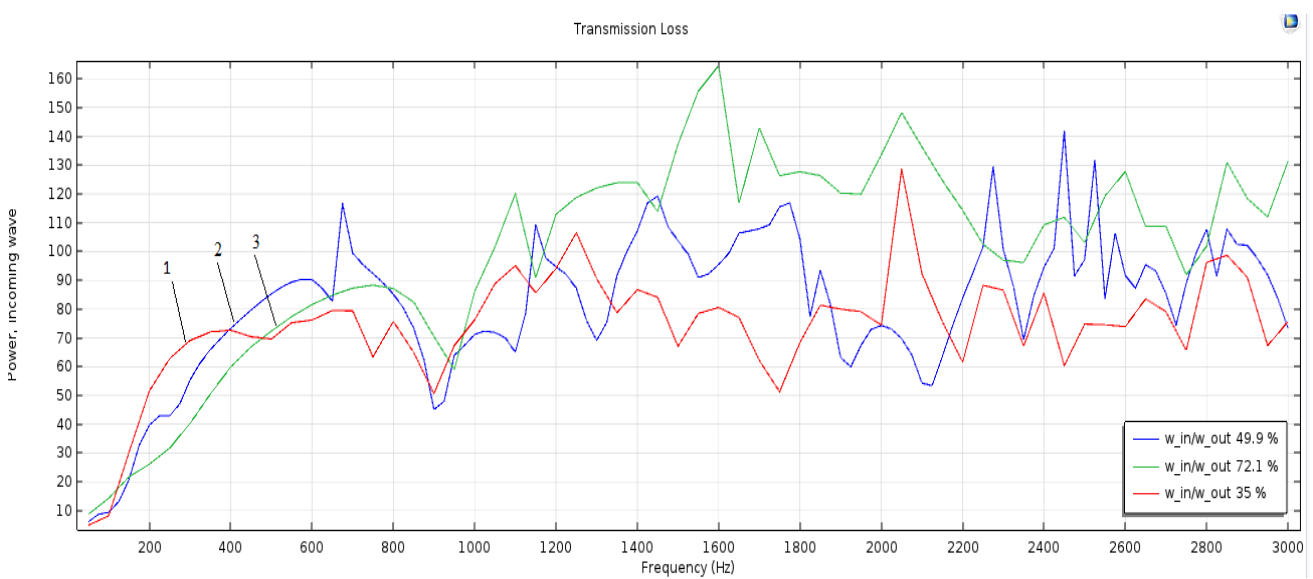

Fig. 5. Transmission losses of the considered noise muffler configurations.

\section{Conclusion}

The numerical calculations carried out in the Comsol Multiphysics software environment made it possible to evaluate the acoustic efficiency of various configurations of a combined noise damper and choose the best one.

\section{References}

1. A.I. Komkin, N.A. Nikiforov, R.M. Simkin On the design of automobile exhaust systems, in Proceedings of the Sixth International congress on sound and vibration, Copenhagen, Denmark (1999)

2. A.I. Komkin, A coust. Phys. 56 (3), 373 (2010)

3. S. G. Smirnov, V. A. Nikolaeva, E. O. Pankova, B ezopasnostczhiznedeyatel @osti, 10, 28 (2017) [in Russian]

4. A.I. Komkin, M .A. M ironov, A .I. Bykov, A coust. Phys. 63 (4), 385 (2017)

5. N. D.Chaynov, V. A.Markov, A. A. Savastenko, Structural Noise and Acoustic Characteristics Improvement of Transport Power Plants, in IOP Conference Series: M aterials Science and Engineering, 327 (2) C. 022069 (2018)

6. R. I. Rakhmatov, V. E. K rutolapov, V. N. Zuzov, V ibroengin. Proc. 25, 135 (2019) 